Résumés des conférences et travaux

\title{
Hagiographie et histoire monastique
}

\author{
Jean-Loup Lemaitre
}

\section{OpenEdition \\ Journals}

Édition électronique

URL : https://journals.openedition.org/ashp/714

DOI : 10.4000/ashp.714

ISSN : 1969-6310

Éditeur

Publications de l'École Pratique des Hautes Études

\section{Édition imprimée}

Date de publication : 1 octobre 2009

Pagination : 213-216

ISSN : 0766-0677

Référence électronique

Jean-Loup Lemaitre, " Hagiographie et histoire monastique ", Annuaire de l'École pratique des hautes études (EPHE), Section des sciences historiques et philologiques [En ligne], 140 | 2009, mis en ligne le 16 octobre 2009, consulté le 06 juillet 2021. URL : http://journals.openedition.org/ashp/714 ; DOI : https://doi.org/10.4000/ashp.714

Ce document a été généré automatiquement le 6 juillet 2021

Tous droits réservés : EPHE 


\title{
Hagiographie et histoire monastique
}

\author{
Jean-Loup Lemaitre
}

\section{Coutumes et statuts : les constitutions des chanoines du Latran auX XIII ${ }^{\mathrm{e}}$ et XIV ${ }^{\mathrm{e}}$ siècles}

1 Le choix du thème a été motivé par la publication simultanée en 2006 des statuts du chapitre de Saint-Jean de Latran, ceux du chapitre régulier par Jochen Johrendt: « Die Statuten des regulierten Laterankapitels im 13. Jahrhundert. Mit einer Edition des Statuten Gregors IX. (1228) und Nikolaus'IV. (1290) ", dans Quellen und Forschungen aus Italienischen Archiven und Bibliotheken, t. 86 (2006), p. 95-143, puis ceux du chapitre sécularisé par Boniface VIII par Louis Duval-Arnould, « Les constitutions de Grégoire XI pour le chapitre du Latran (1369-1373) », dans Rivista di storia della Chiesa in Italia, t. 60 (2006), p. 405-450.

2 La taille même des originaux conservés à Rome dans les archives de Saint-Jean-deLatran, Q.1.A.3 pour les statuts de Grégoire IX de $1228(762 / 770 \times 609 / 620 \mathrm{~mm})$, Q.7.A.1 pour ceux de Nicolas IV de $1290(380 / 385 \times 800 / 810 \mathrm{~mm})$, et surtout Q.8.E.1 pour ceux de Grégoire XI, de $1373(1095 \times 830 \mathrm{~mm})$, de surcroît détériorés, ne nous a pas permis de faire travailler directement les auditeurs sur les reproductions des originaux et nous a forcé de nous contenter des éditions proposées par J. Johrendt et L. Duval-Arnould. S'il n'a pas été possible d'évoquer à travers des exemples concrets les productions de la chancellerie pontificale, nous avons pu en contrepartie procéder à l'examen des méthodes d'édition allemandes et françaises en la matière, qui divergent notablement quant à l'emploi des capitales, au système de ponctuation, mais aussi, pour l'édition de Johrend, quant au choix des variantes.

3 Avant d'aborder le texte même de ces statuts, il nous a semblé utile d'en présenter le cadre: le chapitre du Latran ${ }^{1}$. On sait que Constantin avait donné au pape Melchiade (311-314) les locaux de la caserne des Equites singulares, avec une partie de la propriété du sénateur Palutius Lateranus, dont les biens avaient été autrefois 
confisqués pour le domaine impérial. Le pape y fait alors construire une grande basilique dite " du Latran ", placée sous le vocable du Sauveur puis, dès le IV siècle, de saint Jean-Baptiste et de saint Jean l'Évangéliste. Ces bâtiments sont détruits par un tremblement de terre en 896 et Serge III (904-911) fait reconstruire la basilique. Le Latran est alors la première résidence des papes. Un incendie détruit le patriarchat et la basilique en 1308: les bâtiments sont reconstruits par Clément V (1305-1314) puis complétés par Urbain V (1362-1370). Avec le retour de la papauté à Rome, après son séjour avignonais (1377), la Curie s'installe au Vatican. Les bâtiments sont considérablement modifiés par les papes des Temps modernes : Sixte Quint (1585-1590) fait construire un nouveau palais pontifical par D. Fontana, dont Paul V Borghese fait la résidence des chanoines, et Innocent $\mathrm{X}$ fait restaurer la basilique par Borromini. Une nouvelle façade est construite en 1735. Le presbiterium est restauré sous Léon XIII. Il ne reste plus du monastère de chanoines réguliers qui a assuré le fonctionnement liturgique de la basilique, que le cloître cosmatesque réalisé par les Vasselleto, père et fils, sous les pontificats d'Honorius III et d'Honorius IV (1216-1254).

4 Les origines de la vie communaire sont encore mal connues, faute de sources. On sait qu'il y a aux $\mathrm{VIII}^{\mathrm{e}}-\mathrm{X}^{\mathrm{e}}$ siècles, un monasterium Lateranense, Saint-Pancrace au Latran (situé à l'emplacement du cloître actuel), qui remonterait à la fin $d u$ vie siècle et qui est évoqué par Grégoire le Grand : fondé par les moines du Mont-Cassin, il était alors dirigé par Valentinianus, un disciple de saint Benoît (Dialogues, II, prol. 2). Paul Diacre (Histoire des Lombards, IV, 17) ne parle pas explicitement du monastère. Ce monastère, qui n'a plus de moines au viII ${ }^{\mathrm{e}}$ siècle, est rénové par Grégoire III (731-741), qui y installe une nouvelle communauté et reconstitue son temporel (Liber pontificalis, I, 419). Adrien I $^{\text {er }}$ (772-795) décide que les moines de Saint-Pancrace alterneront avec ceux de SaintHonoré pour le service liturgique de la basilique (Liber pont., I, 506). On a examiné l'ensemble des mentions connues concernant les trois monastères qui sont à l'origine du chapitre : Saint-Pancrace, Saint-Honoré (Saint-André et Saint-Barthélemy) et SaintÉtienne.

5 À partir du XI ${ }^{\mathrm{e}}$ siècle, le service liturgique de la basilique est assuré par des chanoines réguliers (mise en application des synodes d'Aix, 816-817). En 1050, le pape Léon IX concède aux chanoines les fonts baptismaux et les oratoires de diverses églises romaines : c'est la première mention explicite des chanoines du Latran (Kehr, Italia pontificia, I, 25).

6 Nous avons par ailleurs examiné l'ensemble des reliques conservées au Latran (basilique et Sancta Sanctorum), à travers les inventaires anciens, les listes d'indulgences et les authentiques du Sancta Sanctorum ${ }^{2}$. Grâce à l'obligeance de $\mathrm{M}^{\mathrm{gr}}$ Louis DuvalArnould, élève diplômé de la Section, chanoine français et archiviste du Latran, nous avons pu étudier sur photographies la Tabula magna, double panneau de mosaïque exécuté en 1291, situé actuellement dans le passage de la sacristie, dont la première partie donne un état des reliques de la basilique. On ne dénombre pas moins de cent quatorze numéros dans les inventaires anciens, reliques conservées sur le maitre-autel, dans le chœur, sous la voûte, dans la sacristie et dans les chapelles, dont celle du Sancta Sanctorum, d'importance diverse. Certaines sont toujours vénérées, comme le chef de sainte Agnès, mentionné dans l'inventaire du diacre Jean dans les années 1159-1181 et transporté dans l'église de Sainte-Agnès in Agone en 1905. De très nombreuses reliques concernent le Christ et cela nous a permis de rappeler la typologie de ces reliques particulières. 


\section{Les statuts de Grégoire IX.}

7 Grégoire IX (le cardinal Hugolin, des comtes de Segni) a été élu pape le 19 mars 1227. Il meurt le 22 août 1241 et est inhumé à Saint-Pierre. Il est le premier pape à créer des cardinaux non italiens et il développe le rôle de la Curie. Il publie le 12 avril 1231 la bulle Parens scientiarum, qui est considérée comme le point de départ de l'université de Paris et, entre 1235 et 1237, des statuts pour les moines noirs, Statuta ordinis nigri, en 54 articles $^{3}$.

8 Les statuts pour le chapitre du Latran sont donnés le 3 février 1228, sous la forme d'un privilège solennel, avec grande date (avec Rota et Benevalete, mais la bulle est perdue). Ils reprennent en fait des statuts promulgués par Honorius III en 1216 (perdus). Le pape rappelle que les religieux suivent l'ordo canonicus selon la règle de saint Augustin et confirme les possessions et les biens du chapitre, caractéristique des grandes bulles solennelles. Il précise aussi la répartition des oblations faites à l'autel majeur, où sont conservés les chefs de saint Pierre et de saint Paul. La bulle énonce toutes les églises appartenant au chapitre, son temporel, tant dans la ville et diocèse de Rome que dans les diocèses voisins. Toutes ces églises ont été successivement examinées, ce qui a permis à l'auditoire de se familiariser un tant soit peu avec les mé-thodes de la topographie historique, en utilisant les pouillés du Latium (Rationes decimarum), mais aussi avec le mode de fonctionnement d'une communauté regulière. Seuls les derniers paragraphes de ces statuts évoquent la pratique liturgique, le service étant assuré à tour de rôle par les cardinaux évêques, avec le concours du chapitre. Ils abordent aussi la question de l'élection du prieur du chapitre qui doit être choisi prioritairement parmi les religieux de la communauté, sinon parmi ceux d'une autre communauté religieuse, s'il n'y en a pas d'idoine, avec le consentement du pontifie romain. On fixe aussi les règles d'accueil dans la communauté, qui peut recevoir tous les laïcs fuyant le siècle, sauf s'ils sont interdits ou excommuniés. Personne ne pourra par ailleurs prononcer contre le prieur et les chanoines une peine de suspense ou d'excommunication, mais aucun chanoine profès ne pourra quitter la communauté sans l'accord du prieur. L'examen des souscriptions a permis d'évoquer la terminologie des cardinaux au Moyen Âge et divers points de prosopographie ecclésiastique.

\section{Les statuts de Nicolas IV.}

Le franciscain Girolamo Masci, qui avait succédé à saint Bonaventure comme général de l'ordre, cardinal du titre de Sainte-Pudentienne, est élu pape le 22 février 1288. Il meurt le 4 avril 1292 et est inhumé à Sainte-Marie-Majeure. Il promulgue à son tour des statuts pour le chapitre du Latran, le 7 mai 1290. Il ne reprend pas l'énoncé des biens, comme l'avait fait Grégoire IX, mais donne des directives originales, plus concrètes, concernant les officiers du chapitre, désignés chaque année par le prieur et les chanoines: chambrier, vice-chambrier, sacriste, vestiaire, grénetier, infirmier, trésorier, ordinaire, ce dernier posant problème, car il n'est pas connu autrement et ne se retrouve pas dans les constitutions de Grégoire XI. Seuls les dix premiers articles de ces statuts ont pu être examinés à la fin de l'année scolaire. 


\section{Les bibliothèques monastiques et canoniales : livres et inventaires.}

10 Nous avons poursuivi l'étude méthodologique des inventaires des bibliothèques médiévales, en essayant de montrer aux auditeurs, en dehors de leur intérêt intrinsèque, en particulier pour l'étude de la diffusion des textes, quels pouvaient être leur apport pour l'étude de la vie culturelle et intellectuelle, mais aussi de la pratique religieuse, dans les communautés monastiques et canoniales du Moyen Âge. Nous avons continué cette année la lecture et l'analyse d'inventaires d'abbayes anglaises, dont les textes ont été publiés dans R. Sharpe, J.P. Carley, R. M. Thompson et A.G. Watson, English Benedictines Libraries. The shorters Catalogues, Londres, 1996 (Corpus of British Medieval Library Catalogues, 4), à partir des photographies de ces catalogues (pl. 3-4 et 7-8), qui ne couvrent, dans les deux cas, qu'une partie de ces inventaires:

1. Bury St Edmunds,dans le Suffolk : fin du XII ${ }^{e}$ siècle, Cambridge, Pembrooke College, MS. 47, f. 118r-118v (entrées 62-95 et 96-137) [B 13].

2. Glastonbury, dans le Sommerset : 1247, Cambridge, Trinity College, MS. R.5.33, f. 103v-103r (entrées 93-116 et 167-253) [B 39].

11 Il s'agit dans les deux cas d'inventaires comptant parmi les plus importants des inventaires anglais médiévaux d'abbayes de moines noirs: 261 entrées pour Bury St Edmunds et surtout 402 pour Glastonbury. Cette dernière abbaye en particulier a laissé d'autres inventaires : liste des livres copiés par Henri de Blois (même ms., f. 23r), 40 entrées ; - livres écrits par le prieur Thomas (même ms., f. 41ra). On a pris également en compte les inventaires postérieurs: livres acquis par l'abbé John of Taunton [1274-1281] (même ms., f. 68r), 24 entrées ; - livres données par l'abbé Walter of Taunton (Cambridge, Trinity College, MS. R.5.16, p. 200), 9 entrées ; - livres donnés par Adam de Sodbury [1323-1334] (même ms., p. 204), 4 entrées ; - et surtout liste des livres acquis par l'abbé Walter de Monington [1342-1375] (Cambridge, Trinity College, MS. R.516, p. 246-250), 99 entrées, sans compter les relevés faits par Leland entre 1536-1540, 44 entrées, et par John Bale, vers 1550, avec 69 entrées. Ces données ont permis, à partir des titres mentionnés dans les deux inventaires étudiés, de voir leur traitement éventuel dans les autres inventaires.

\section{NOTES}

1. Le palais a fait l'objet d'une monumentale étude de Ph. Lauer, Le palais du Latran. Étude historique et archéologique, Paris, 1911, in-fol., IV-648 p., XXXV pl. et un plan hors-texte.

2. N. B. Miedema, Die römischen Kirchen im Spätmittelalter nach den "Indugentiae ecclesiarum urbis Romae”, Tübingen, 2001 ; Ph. Lauer, Le Trésor du Sancta Sanctorum, Paris, 1906 ; B. Galland, Les authentiques de reliques du Sancta Sanctorum, Vatican, 2004.

3. Éd. L. Auvray, Les registres de Grégoire IX..., t. II, Paris, 1907, p. 317-322. 


\section{RÉSUMÉS}

Programme de l'année 2007-2008: I. Coutumes et statuts: les constitutions des chanoines du Latran aux $\mathrm{XIII}^{\mathrm{e}}$ et $\mathrm{XIV}^{\mathrm{e}}$ siècles. - II. Les bibliothèques monastiques et canoniales: livres et inventaires.

INDEX

Thèmes : Hagiographie et histoire monastique

\section{AUTEUR}

JEAN-LOUP LEMAITRE

Directeur d'études, École pratique des hautes études - Section des sciences historiques et philologiques 